\title{
An in vitro splicing assay reveals the pathogenicity of a novel intronic variant in ATP6V0A4 for autosomal recessive distal renal tubular acidosis
}

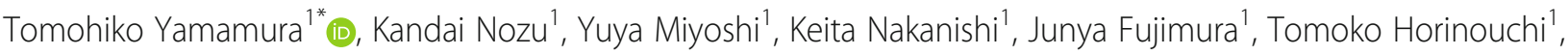 \\ Shogo Minamikawa', Nobuo Mori ${ }^{2}$, Rika Fujimaru ${ }^{3}$, Koichi Nakanishi ${ }^{4}$, Takeshi Ninchoji ${ }^{1}$, Hiroshi Kaito ${ }^{1}$, \\ Taniguchi-Ikeda Mariko ${ }^{1}$, Ichiro Morioka', Masafumi Matsuo ${ }^{5}$ and Kazumoto lijima'
}

\begin{abstract}
Background: Autosomal recessive distal renal tubular acidosis (dRTA) is a rare hereditary disease caused by pathogenic variants in the ATP6VOA4 gene or ATP6V1B1 gene, and characterized by hyperchloremic metabolic acidosis with normal anion gap, hypokalemia, hypercalciuria, hypocitraturia and nephrocalcinosis. Although several intronic nucleotide variants in these genes have been detected, all of them fell in the apparent splice consensus sequence. In general, transcriptional analysis is necessary to determine the effect on function of the novel intronic variants located out of splicing consensus sequences. In recent years, functional splicing analysis using minigene construction was used to assess the pathogenicity of novel intoronic variant in various field.

Methods: We investigated a sporadic case of dRTA with a compound heterozygous mutation in the ATP6VOA4 gene, revealed by next generation sequencing. One variant was already reported as pathogenic; however, the other was a novel variant in intron 11 (c.1029 + 5G > A) falling outside of the apparent splicing consensus sequence. Expression of ATP6VOA4 was not detected in peripheral leukocytes by RT-PCR analysis. Therefore, an in vitro functional splicing study using minigene construction was conducted to analyze the splicing pattern of the novel variant.
\end{abstract}

Results: A minigene assay revealed that the novel intronic variant leads to a 104 bp insertion immediately following exon 11. In addition, this result was confirmed using RNA extracted from the patient's cultured leukocytes.

Conclusion: These results proved the pathogenicity of a novel intronic variant in our patient. We concluded that the minigene assay is a useful, non-invasive method for functional splicing analysis of inherited kidney disease, even if standard transcriptional analysis could not detect abnormal mRNA.

Keywords: Autosomal recessive distal renal tubular acidosis, ATP6VOA4, Minigene, Splicing assay

\section{Background}

Renal tubular acidosis (RTA) is characterized by normal serum anion gap or hyperchloremic metabolic acidosis and four types of RTA can be distinguished on the basis of clinical, pathophysiological, and molecular differences. $[1,2]$. Hereditary distal renal tubular acidosis (dRTA) is caused by mutations in genes that encode three proteins

\footnotetext{
* Correspondence: tomohiko@med.kobe-u.ac.jp

${ }^{1}$ Department of Pediatrics, Kobe University Graduate School of Medicine,

7-5-1 Kusunokicho, Chuo, Kobe, Hyogo 6500017, Japan

Full list of author information is available at the end of the article
}

expressed in $\alpha$-intercalated cells of the collecting duct, namely, the $\mathrm{B} 1$ or $\mathrm{A} 4$ subunits of $\mathrm{V}$-ATPase and the kidney $\mathrm{Cl}^{-} / \mathrm{HCO}^{-}$exchanger (kAE1) [2-5]. ATP6V1B1 and ATP6VOA4 encode the B1 and a4 subunits of the V-ATPase, respectively. Pathogenic variants in these genes interrupt the function of V-ATPase and cause autosomal recessive dRTA and sensorineural hearing loss.

In recent years, it was reported that next generation sequencing (NGS) is a useful diagnostic tool of the genetic analysis for dRTA and many pathogenic variants including intronic variants have been detected so far [6]. However, 
fewer than 10 intronic variants have been identified in the ATP6VOA4 gene and all these variants are located in apparent splicing consensus sequences (HGMD Professional 2016.4) [7]. In general, transcript analysis is necessary for variants located outside of the splicing consensus sequence. However, this technique is difficult for dRTA because the ATP6VOA4 transcript expression level is very low in peripheral blood leukocytes, usually the only samples available from patients.

Recently, functional splicing analysis using minigene construction has been used to investigate novel intronic variants in various inherited diseases and revealed its usefulness [8-12]. However, in the field of inherited kidney diseases, there is very little information about this minigene assay $[13,14]$.

\section{Methods}

A one-year-old Japanese boy was referred to our hospital for further evaluation of failure to thrive and suspected renal tubular acidosis (RTA) by the family doctor. Blood examination showed hyperchloremic non-gap metabolic acidosis and hypokalemia (chloride: $110 \mathrm{meq} / \mathrm{L}$; blood pH: 7.25; bicarbonate: $11.5 \mathrm{mmol} / \mathrm{L}$; potassium: $3.1 \mathrm{meq} / \mathrm{L}$ ). Urinalysis showed high urine $\mathrm{pH}$ (7.0), positive urine anion gap $(15 \mathrm{mmol} / \mathrm{L})$, hypercalciuria, hyperkaliuria and low molecular proteinuria (calcium/creatinine ratio: $0.38 \mathrm{~g} / \mathrm{gcre}$ (normal: below $0.2 \mathrm{~g} /$ gcre); potassium/creatinine ratio 10.5 (normal: below 2); beta-2-microgloblin: 79,639 $\mu \mathrm{g} / \mathrm{L}$ (normal: below $400 \mu \mathrm{g} / \mathrm{L}$ )). The calculated transtubular potassium gradient (TTKG) was 5.5 and the fractional excretion of potassium (FEK) was $9.8 \%$. Abdominal ultrasonography detected bilateral renal calcinosis. Furosemide and $\mathrm{NaHCO}_{3}$ load test indicated that clinical diagnosis was dRTA. Audiometry screening test did not detect sensorineural deafness.

After obtaining informed consent from the parents of the proband, we performed targeted sequencing using next generation sequencing (NGS). NGS samples were prepared using a HaloPlex Target Enrichment System Kit (Agilent Technologies, Santa Clara, CA, USA) according to the manufacturer's instructions to capture 17 genes (Additional file 1: Table S1), including genes associated with renal tubular disorder. Amplified target libraries were sequenced using MiSeq (Illumina, San Diego, CA, USA) and analyzed with SureCall (v.3.0; Agilent Technologies). The ATP6V0A4 reads were mapped to the human reference sequence NM_020632.2.

In this study, hybrid minigene constructs were created by inserting a test sequence fragment consisting of exon 11 and exon 12 and its flanking introns into the multicloning site within an intervening intron between two exons (exon A and B) of the minigene construct (H492), built in the pcDNA 3.0 mammalian expression vector (Invitrogen, Carlsbad, CA) (Additional file 1: Figure S1)
[14]. These hybrid minigenes were transfected into HEK293T cells and HeLa cells for splicing assays as described elsewhere $[10,11]$. Both HEK293T and HeLa cells were obtained from the Cell Bank, Riken Bio Resource Center (Tsukuba, Japan). Total RNA was reversetranscribed into cDNA and the PCR was performed using specific primers as previously described [10] (Additional file 1: Figure S1). PCR products were analyzed by means of electrophoresis and direct sequencing.

In addition, patient's leukocyte culture was performed to increase the total RNA amount for detecting splicing abnormalities. The detailed method of cultured leukocyte is as follows; $8.5 \mathrm{~mL}$ of patient's peripheral blood is mixed to $1.5 \mathrm{~mL}$ of Acid Citrate Dextrose solution (Becton-Dickinson \& Company, Franklin Lakes, NJ) at room temperature. Transfer a blood and Acid Citrate Dextrose solution mix into a $50 \mathrm{ml}$ tube and dilute blood with an equal volume of PBS and mix gently. Add $5 \mathrm{~mL}$ of FicollPaque PREMIUM (GE Healthcare, UK) to a $15 \mathrm{~mL}$ tube. Gently overlay $5 \mathrm{~mL}$ of the diluted blood onto $5 \mathrm{~mL}$ of Ficoll without disturbing the interface. Centrifuge at $400 \times \mathrm{g}$ for $30 \mathrm{~min}$ at $25^{\circ} \mathrm{C}$ and carefully collect the whitish layer in the upper-middle interface into a $15 \mathrm{~mL}$ tube. Add 6 times of volume of PBS and mix gently and centrifuge at $200 \times \mathrm{g}$ for $10 \mathrm{~min}$ at $25{ }^{\circ} \mathrm{C}$. Aspirate the supernatant and add $2 \mathrm{~mL}$ of KBM-502 medium (KOHJIN $\mathrm{BIO}, \mathrm{JP})$ to the pellet. Incubate cells at $5 \% \mathrm{CO}_{2}$ incubator at $37^{\circ} \mathrm{C}$ for 1 week and correct total RNA.

\section{Results}

\section{Mutational analysis}

Targeted sequencing and direct sequencing revealed a novel heterozygous variant of one base substitution at the 5th base of intron $11($ c. $1029+5 \mathrm{G}>\mathrm{A})$ and a previously reported variant of one base substitution in exon 22 (c.2420G > A, p.Arg807Gln [15]) in the ATP6VOA4 gene. The variants were derived from the paternal and maternal alleles, respectively (Fig. 1a). RNA was extracted from peripheral blood leukocytes for RT-PCR analysis, however the ATP6VOA4 transcript failed to amplify because of its low expression.

\section{In vitro splicing assay}

The RT-PCR product containing the expected exon 11 exon 12 sequence between the cassette exons $\mathrm{A}$ and $\mathrm{B}$ was obtained from the minigene encoding the wild-type sequence (Fig. 2a-b). In contrast, the product from the hybrid minigene containing patient sequence with the intronic variant showed a 104 bp sequence insertion between exon 11 and exon 12 .

\section{RT-PCR using RNA extracted from cultured leukocytes}

After finding the splicing abnormality using the minigene construct, we used RT-PCR analysis on RNA extracted 


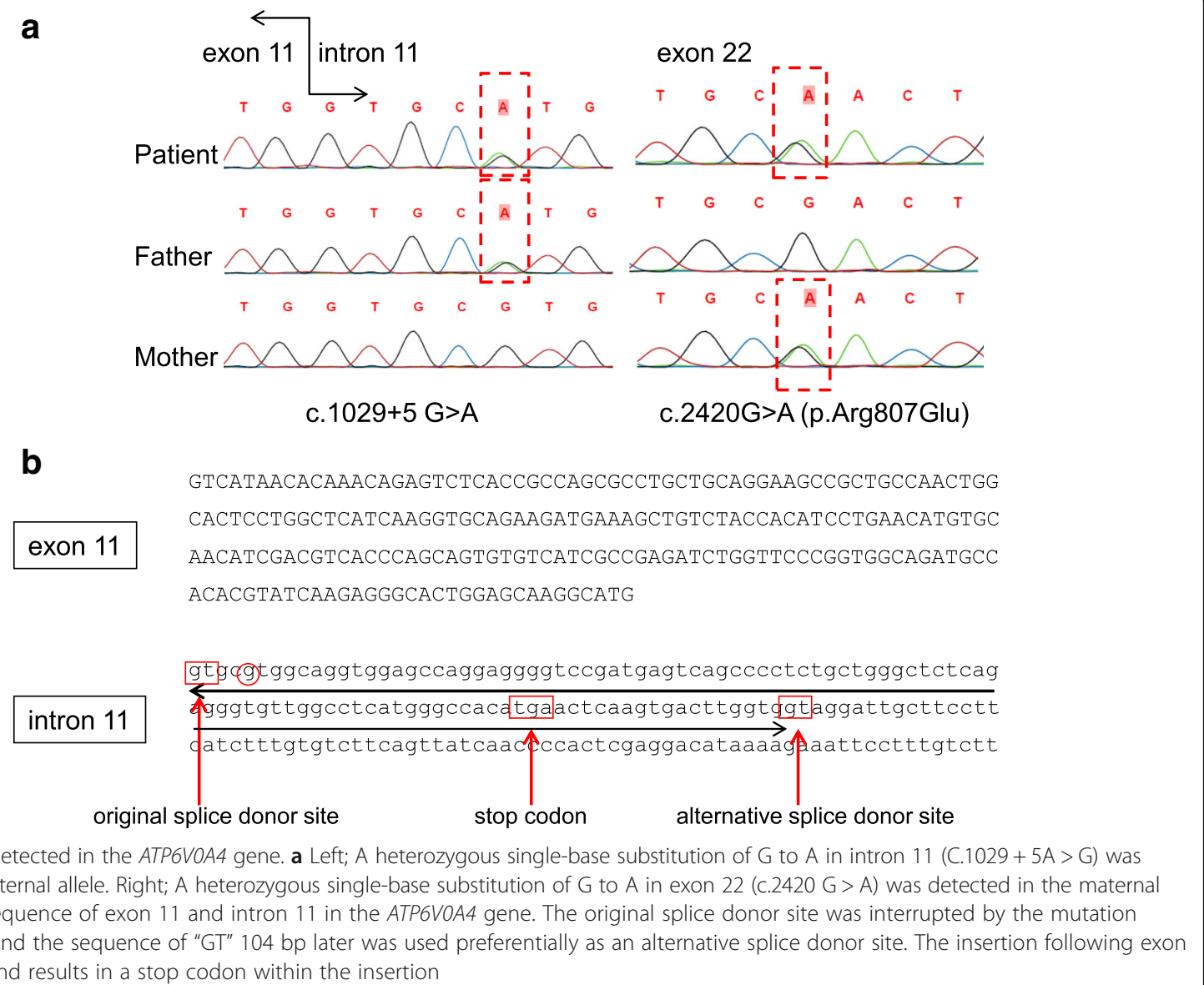

from patient cultured lymphocytes to confirm the result. RT-PCR showed two bands: one the same size as the control, and the other larger, consistent with the 104 bp product detected in the minigene assay (Fig. 3). Sequencing of the larger product confirmed the $104 \mathrm{bp}$ insertion following exon 11. This insert sequence created a stop codon.

\section{Discussion}

To assess the biological effect of intronic variants that do not fall in splicing consensus sequences, transcript analysis is necessary. However, there are several reasons why this may not be successful. First, abnormal transcripts can be hard to amplify by RT-PCR because of the influence of nonsense-mediated mRNA decay (NMD) for the products of truncating variants. Second, transcripts extracted from tissues other than blood leukocytes are not always available in various diseases and targeted transcript expression may be low in leukocytes. To resolve these problems, we conducted an in vitro splicing assay using hybrid minigene construction, which revealed an alternative splice donor site that was used in preference to the original site (Fig. 1b). This finding indicated that the c. $1029+5 \mathrm{~A}>\mathrm{G}$ variant was pathogenic.
To confirm this result, we used mRNA extracted from cultured leukocytes and, fortunately, we could detect the same 104 bp insertion.

We were able to detect the abnormal transcript in the patient's cultured leukocytes in this study. However, this method takes a lot of effort, including obtaining fresh samples, which is not possible for all cases. Although renal biopsy specimens could be used as a source of mRNA in inherited kidney diseases, renal biopsy is invasive and not necessary for most inherited kidney diseases, including dRTA. Alternatively, urine sediments can be used for genetic analysis in inherited kidney diseases $[14,16]$. However, in this case, we also failed to obtain mRNA from patient's urine because of polyuria, a symptom of RTA, which results in diluted urine.

Here we report, for the first time, the use of an in vitro splicing assay using minigene construction, in the characterization of a mutation in dRTA, although this assay has previously been conducted for several diseases, including some inherited kidney diseases [10$14,17,18]$. Some reports used this analysis because they could not obtain mRNA from kidney specimens [17]. This time, we could confirm the splicing 

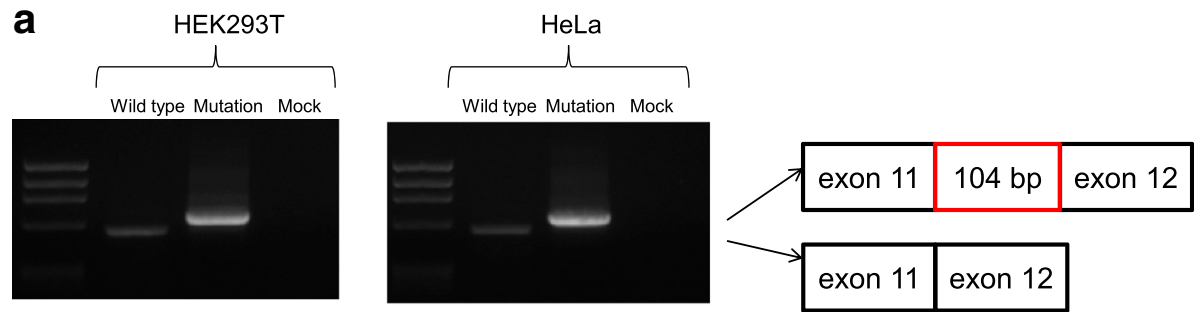

b

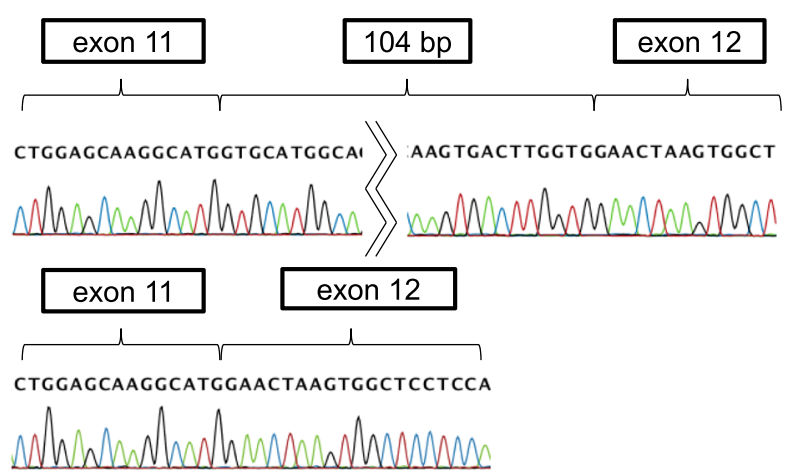

Fig. 2 In vitro splicing assay using hybrid minigene construct. a RT-PCR amplified products of hybrid minigene transcripts. As shown in the gel file, a comparatively small band was present in the wild type construct both in HEK293T and HeLa cells. In contrast, in the minigene derived from the patient sequence, only a relatively large band was detected in both cell lines, which corresponds to a 104 bp insertion between exon 11 and exon12. b The RT-PCR product containing the 104 bp insertion between exon 11 and exon 12 was obtained from the minigene encoding the intronic mutation (b top). On the other hand, the product containing exon 11 and exon 12 could be obtained from the hybrid minigene encoding the wild type genomic DNA (b bottom)

abnormality using both the patient's sample and minigene construction.

In silico analysis is generally used to estimate the pathogenicity of novel intronic variants. In this case, we tried to predict the alternative splice donor site using Human Splicing Finder 3.0 (http://www.umd.be/HSF3/) [19]; however, this variant was predicted as having "probably no impact" on splicing (Additional file 1: Figure S2). This shows that in silico analysis does not always predict accurately. Therefore, the in vitro functional splicing assay using a minigene is an attractive tool for cases such as the one described here.

\section{Conclusions}

In conclusion, our study demonstrates that the minigene assay is a useful, non-invasive method for functional splicing analysis of inherited kidney disease, even if standard transcriptional analysis could not detect abnormal mRNA. This analytical assay could be adapted for other inherited kidney diseases.

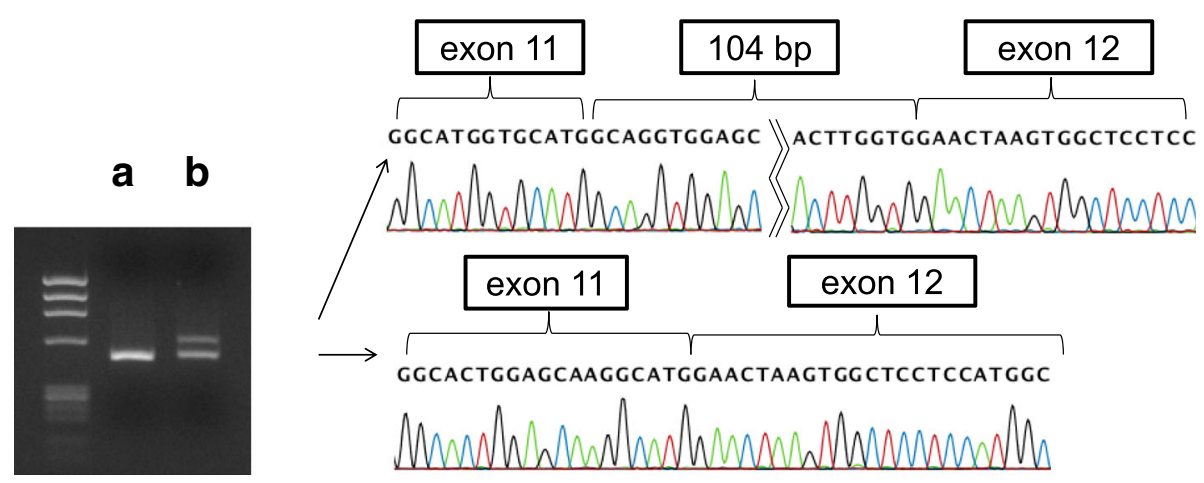

Fig. 3 Direct sequencing results of cDNA after PCR. The control leukocyte sample shows only one normal band (a), the sample extracted from lymphocyte culture of the patient (b) shows two bands, one the same size as the control sample and the other including a 104 bp insertion sequence 


\section{Additional file}

Additional file 1: Table S1. List of genes analyzed by target sequencing in this study. Figure $\mathbf{S 1}$. Diagram of hybrid minigene construct. Figure S2. The result of alternative splicing pattern prediction using Human Splicing Finder Ver.3.0. (DOCX 296 kb)

\section{Abbreviations}

dRTA: Distal renal tubular acidosis; RT-PCR: Reverse-transcription PCR

\section{Funding}

This study was supported by a grant from the Ministry of Health, Labour, and Welfare (Japan) for Research on Rare Intractable Diseases in the Kidney and Urinary Tract (H24-nanchitou (nan)-ippan-041 to KI) in the "Research on Measures for Intractable Diseases" Project, and a Grant-in-Aid for Scientific Research (KAKENHI) from the Ministry of Education, Culture, Sports, Science and Technology of Japan (Subject ID: 16K19642 to TY and 25893131 to KaN and 26293203 to $\mathrm{KI}$ )

\section{Availability of data and materials}

The datasets used and analyzed during the current study is available from the corresponding author on reasonable request.

\section{Authors' contributions}

NM and RF analyzed the clinical data including load tests and validated consistency between the clinical data and the result of genetic analysis; KaN and MM conceived the idea; TY, KeN and YM collected data and wrote the manuscript; JF, TH and SM carried out the next generation sequencing, data analysis, variant interpretation and reporting. TY, TN and HK conducted functional splicing analysis using minigene construction. KoN, TIM, IM and KI critically reviewed the manuscript. All authors read and approved the final version of the manuscript.

\section{Ethics approval and consent to participate}

All procedures were approved by the Institutional Review Board (IRB) of Kobe University Graduate School of Medicine (IRB number: 301). Signed informed consent was obtained from the parents of the proband.

\section{Consent for publication}

Signed informed consent to publish was obtained from the parents of the proband.

\section{Competing interests}

The authors declare that they have no competing interests.

\section{Publisher's Note}

Springer Nature remains neutral with regard to jurisdictional claims in published maps and institutional affiliations.

\section{Author details}

${ }^{1}$ Department of Pediatrics, Kobe University Graduate School of Medicine, 7-5-1 Kusunokicho, Chuo, Kobe, Hyogo 6500017, Japan. ${ }^{2}$ Nakano Children's Hospital, 4-13-17 Shinmori, Asahi, Osaka 5350022, Japan. ${ }^{3}$ Department of Pediatrics, Osaka City General Hospital, 2-13-22 Miyakojimahondori, Miyakojima, Osaka 5340021, Japan. ${ }^{4}$ Department of Child Health and Welfare (Pediatrics), Graduate School of Medicine, University of the Ryukyus, 207 Uehara, Nishihara, Nakagami 9030125, Japan. ${ }^{5}$ Department of Physical Therapy, Faculty of Rehabilitation, Kobe Gakuin University, 518 Arise, Ikawadani, Nishi, Kobe 6512180, Japan.

Received: 24 July 2017 Accepted: 23 November 2017 Published online: 04 December 2017

\section{References}

1. Gil-Pena H, Mejia N, Santos F. Renal tubular acidosis. J Pediatr. 2014;164:691-8. e691

2. Santos F, Gil-Pena H, Alvarez-Alvarez S. Renal tubular acidosis. Curr Opin Pediatr. 2017;29:206-10.
3. Batlle D, Haque SK. Genetic causes and mechanisms of distal renal tubular acidosis. Nephrol Dial Transplant. 2012;27:3691-704.

4. Both $T$, Zietse R, Hoorn EJ, van Hagen PM, Dalm VA, van Laar JA, van Daele PL. Everything you need to know about distal renal tubular acidosis in autoimmune disease. Rheumatol Int. 2014;34:1037-45.

5. Rodriguez Soriano J. Renal tubular acidosis: the clinical entity. J Am Soc Nephrol. 2002;13:2160-70.

6. Gomez J, Gil-Pena H, Santos F, Coto E, Arango A, Hernandez O, Rodriguez J, Nadal I, Cantos V, Chocron S, et al. Primary distal renal tubular acidosis: novel findings in patients studied by next-generation sequencing. Pediatr Res. 2016;79:496-501.

7. Stenson PD, Ball EV, Mort M, Phillips AD, Shiel JA, Thomas NS, Abeysinghe S, Krawczak M, Cooper DN. Human gene mutation database (HGMD): 2003 update. Hum Mutat. 2003;21:577-81.

8. Niba ET, Nishida A, Tran VK, DC V, Matsumoto M, Awano H, Lee T, Takeshima Y, Nishio H, Matsuo M. Cryptic splice activation but not exon skipping is observed in minigene assays of dystrophin c.9361+1G>A mutation identified by NGS. J Hum Genet. 2017;

9. Nishida A, Oda A, Takeuchi A, Lee T, Awano H, Hashimoto N, Takeshima Y, Matsuo M. Staurosporine allows dystrophin expression by skipping of nonsense-encoding exon. Brain and Development. 2016;38:738-45.

10. Thi Tran HT, Takeshima Y, Surono A, Yagi M, Wada H, Matsuo M. A G-To-a transition at the fifth position of intron-32 of the dystrophin gene inactivates a splice-donor site both in vivo and in vitro. Mol Genet Metab. 2005:85:213-9.

11. Tran VK, Takeshima Y, Zhang Z, Habara Y, Haginoya K, Nishiyama A, Yagi M, Matsuo M. A nonsense mutation-created intraexonic splice site is active in the lymphocytes, but not in the skeletal muscle of a DMD patient. Hum Genet. 2007;120:737-42

12. Tran VK, Takeshima Y, Zhang Z, Yagi M, Nishiyama A, Habara Y, Matsuo M. Splicing analysis disclosed a determinant single nucleotide for exon skipping caused by a novel intraexonic four-nucleotide deletion in the dystrophin gene. J Med Genet. 2006;43:924-30.

13. Malone AF, Funk SD, Alhamad T, Miner JH. Functional assessment of a nove COL4A5 splice region variant and immunostaining of plucked hair follicles as an alternative method of diagnosis in X-linked Alport syndrome. Pediatr Nephrol. 2016;

14. Nozu K, lijima K, Kawai K, Nozu Y, Nishida A, Takeshima Y, XJ F, Hashimura Y, Kaito $\mathrm{H}$, Nakanishi $\mathrm{K}$, et al. In Vivo and in vitro splicing assay of SLC12A1 in an antenatal salt-losing tubulopathy patient with an intronic mutation. Hum Genet. 2009;126:533-8.

15. Stover EH, Borthwick KJ, Bavalia C, Eady N, Fritz DM, Rungroj N, Giersch AB, Morton CC, Axon PR, Akil l, et al. Novel ATP6V1B1 and ATP6V0A4 mutations in autosomal recessive distal renal tubular acidosis with new evidence for hearing loss. J Med Genet. 2002;39:796-803.

16. Igarashi T, Inatomi J, Ohara T, Kuwahara T, Shimadzu M, Thakker RV. Clinical and genetic studies of CLCN5 mutations in Japanese families with Dent's disease. Kidney Int. 2000;58:520-7.

17. Bergmann C, Frank V, Kupper F, Schmidt C, Senderek J, Zerres K. Functional analysis of PKHD1 splicing in autosomal recessive polycystic kidney disease. J Hum Genet. 2006;51:788-93.

18. Takeuchi Y, Mishima E, Shima H, Akiyama Y, Suzuki C, Suzuki T, Kobayashi T, Suzuki Y, Nakayama T, Takeshima Y, et al. Exonic mutations in the SLC12A3 gene cause exon skipping and premature termination in Gitelman syndrome. J Am Soc Nephrol. 2015;26:271-9.

19. Desmet FO, Hamroun D, Lalande M, Collod-Beroud G, Claustres M, Beroud C. Human splicing finder: an online bioinformatics tool to predict splicing signals. Nucleic Acids Res. 2009;37:e67. 